\title{
The Effect of Volume Controlled and Pressure Controlled Ventilation Modes on Cerebral Oximetry and Blood Gas Status in Laparoscopic Cholecystectomy, A Randomized Controlled Trial
}

\section{Emre Badur}

Anesthesiology and Reanimation Department, Sisli Hamidiye Etfal Training and Researche Hospital, University of Health Sciences, Istanbul, Turkey

\section{Mustafa Altınay ( $\nabla$ m_altinay@yahoo.com )}

Anesthesiology and Reanimation Department, Sisli Hamidiye Etfal Training and Researche Hospital, University of Health Sciences, Istanbul, Turkey

\section{Pınar Sayın}

Anesthesiology and Reanimation Department, Sisli Hamidiye Etfal Training and Researche Hospital, University of Health Sciences, Istanbul, Turkey

\section{Ayşe Surhan Çınar}

Anesthesiology and Reanimation Department, Sisli Hamidiye Etfal Training and Researche Hospital, University of Health Sciences, Istanbul, Turkey

\section{leyla türkoğlu}

Anesthesiology and Reanimation Department, Sisli Hamidiye Etfal Training and Researche Hospital, University of Health Sciences, Istanbul, Turkey

\section{tuğba yücel}

Anesthesiology and Reanimation Department, Dr.Sadi KONUK Training and Researche Hospital, University of Health Sciences, Istanbul, Turkey

\section{Research Article}

Keywords: Near infrared spectroscopy, NIRS, cerebral oximetry, abdominal surgery

Posted Date: October 22nd, 2021

DOl: https://doi.org/10.21203/rs.3.rs-871798/v1

License: (a) (i) This work is licensed under a Creative Commons Attribution 4.0 International License. Read Full License 


\section{Abstract}

Background: To compare the volume-controlled and pressure-controlled ventilation modes with near infrared spectroscopy (NIRS) cerebral oximetry and blood gas status in laparoscopic cholecystectomy

Methods: Seventy patients $(n=70)$, who underwent elective laparoscopic cholecystectomy operation were randomized into two groups (volume control ventilation - group V, pressure control ventilation - group P). Demographic data (age, gender, body mass index) and operative data (anesthesia, surgery, and insufflation durations) were recorded. Patients' single derivation electrocardiogram, pulse oximetry, noninvasive arterial pressure, NIRS cerebral oximetry and end-tidal $\mathrm{CO}_{2}$ parameters were recorded. Measurements were done at the start of anesthesia (T0), at the end of intubation (T1), 5 minutes after the insufflation (T2), at the time just before desufflation (T3) and 5 minutes after desufflation (T4).

The patients' heart rate, systolic and diastolic arterial pressure, saturation of pulse oximetry, and NIRS values were recorded for time points. Additionally, arterial gas results and mechanical ventilation parameters were recorded as well.

Results: No significant difference was found in age, sex, body mass index. Operation, anesthesia and insufflation durations were similar for the groups. In Group P, NIRS right T1-2-3 averages and NIRS left T2-3 averages were significantly higher than Group $V(p=0.030, p=0.001, p=0.001, p=0.006, p=0.002$ respectively). In Group P T1-T2-T4, mean peak pressures and mean plateau pressures were significantly lower than Group V ( $p=0.003, p=0.001, p<0.001, p=0.011, p=0.001, p<0.001$ respectively).

Conclusion: Mechanical ventilation that performed in pressure-control ventilation mode is resulted with better tissue oxygenation than volume-control ventilation mode. In pressure-control ventilation mode, peak pressure and plateau pressure were lower.

Registration of study at ClinicalTrials.gov was made at 25/01/2021 with the NCT04723043 number.

\section{Background}

Since the laparoscopic methods have been introduced to the surgical operations, laparoscopic cholecystectomy has become the golden standard in cholelithiasis surgery. ${ }^{1}$ For laparoscopic surgery, carbon dioxide $\left(\mathrm{CO}_{2}\right)$ insufflation is used which increase the intra-abdominal pressure. The arterial oxygenation, the functional residual capacity and the lung compliance will be affected and may be resulted with cardiovascular events. ${ }^{2,3}$

Volume-controlled ventilation (VCV) and pressure-controlled ventilation (PCV) are two mechanical ventilation modes that can be used along with their own advantages and disadvantages. ${ }^{3}$ The VCV needed a pre-determined tidal volume (TV). The risk of lung damage is the main concern. In contrast the PCV avoid from excess respiratory tract pressure which applied to the lung. However TV may become 
unstable. Both techniques previously evaluated if one provide lower respiratory work and better tissue oxygenation. Some studies indicated that PCV is better for arterial and tissue oxygenation. ${ }^{4}$

Along with the arterial gas results, near infrared spectroscopy (NIRS) is used to evaluate the depth of anesthesia by measuring the oxygenation change at the tissue level in the prefrontal cortex. ${ }^{5}$ Although NIRS was used in different surgeries, their use in laparoscopic abdominal surgery is extremely

limited. ${ }^{5,6}$ In the available literature we could not be able to find a study which evaluates the effectiveness of perioperative ventilation modes with the NIRS method.

The aim of present study was to compare VCV and PCV modes with NIRS cerebral oximetry and arterial gas results in laparoscopic cholecystectomy

\section{Materials And Methods}

A prospective, randomized study was conducted in the Sisli Hamidiye Etfal Training and Research Hospital between March and July 2020. Study was started after obtaining approval from the local ethics committee with the approval number: 1496. Registration of study at ClinicalTrials.gov was made at 25/01/2021 with the NCT04723043 number. Informed consent was taken from all patients. All procedures that performed in our study were made in accordance with the ethical standards of the Helsinki declaration (2008).

\section{Sample size calculation and randomization}

Considering the difference in large effect size (effect size=0.8) between the groups, the sample size was calculated as 70 cases in total for $95 \%$ Power, alpha significance level 0.05 . Randomization was done with closed envelopes before the procedure.

\section{Inclusion and exclusion criteria}

For the study period patients that underwent elective laparoscopic cholecystectomy were enrolled for the study. Patients aged between 18 and 65, with American Society of Anesthesiology (ASA) score 1 and 2, body mass index $(\mathrm{BMI})<30 \mathrm{~kg} / \mathrm{m} 2$, were included.

Patients who did not give informed consent, patients who underwent previous thoracic/abdominal surgery, patients who underwent emergency laparoscopic cholecystectomy, patients who have ASA score $\geq 3$, hematocrit value $\leq 30$ and, BMl> $30 \mathrm{~kg} / \mathrm{m} 2$ were excluded. Patients with a history of cardiac, neuromuscular, hepato-renal, endocrine, major pulmonary disease (defined as a decrease in capacity or flow rates below $70 \%$ in pulmonary function tests) were also excluded. Patients who returned to laparotomy for surgical reasons after starting laparoscopically, who developed perioperative hemodynamic instability and who used respiratory mechanics outside the study protocol were excluded from the study. 
The primary outcomes of the study were the cerebral oxygenation measured with NIRS, peak pressure and plateau pressure of the patients in both groups, the secondary findings were the patients' $\mathrm{SpO}_{2}$, endtidal carbon dioxide and partial oxygen pressure in arterial blood gases.

\section{Preoperative care}

All patients underwent standard anesthesia evaluation for the procedure. Premedication was done with $0,07 \mathrm{mg} / \mathrm{kg}$ intravenous midazolam.

\section{Intraoperative care}

Single derivation electrocardiogram, pulse-oximetry, noninvasive arterial pressure and $\mathrm{EtCO}_{2}$ parameters were monitored. NIRS monitoring was performed using a Masimo (Irvine, CA, USA) device. NIRS cerebral probes were placed in the right and left frontal regions. A 20-gauge cannula was inserted into the radial artery. Anesthesia induction is by intravenous administration of $2 \mathrm{mg} / \mathrm{kg}$ propofol, $1 \mathrm{mg} / \mathrm{kg}$ lidocaine, 1.5 $\mathrm{mcg} / \mathrm{kg}$ fentanyl and $0.6 \mathrm{mg} / \mathrm{kg}$ rocuronium bromide. Anesthesia maintenance was done with sevoflurane $\% 2$ and remifentanil $0.15-0.25 \mathrm{mcg} / \mathrm{kg} / \mathrm{hour}$. During the maintenance process, the oxygen-air flow was set to $4 \mathrm{lit} / \mathrm{min}$ and the $\mathrm{FiO}_{2}$ set to $40 \%$. During anesthesia, mechanical ventilation was applied to the patients with a Drager (Medical, Lübeck, Germany) brand device.

Mechanical ventilation settings applied to all patients were adjusted according to ideal body weight. In the $\mathrm{P}$ group, inspiratory pressure $\left(\mathrm{P}_{\text {insp }}\right)$ was set to create a tidal volume of $8 \mathrm{ml} / \mathrm{kg}$ in pressurecontrolled mode, while in the $\mathrm{V}$ group, the tidal volume was set as $8 \mathrm{ml} / \mathrm{kg}$ in the volume-controlled mode. In both groups, the initial respiratory frequency was 12 breaths/minutes, the inspiration/expiration time ratio was $1 / 2, \mathrm{FiO}_{2}$ was $40 \%$, and positive end expiratory pressure (PEEP) was $5 \mathrm{~cm} / \mathrm{H}_{2} \mathrm{O}$. While applying mechanical ventilation in all patients, it was aimed to keep the $\mathrm{EtCO}_{2}$ value between 33-35 $\mathrm{mmHg}$. If the $\mathrm{EtCO}_{2}$ was above $35 \mathrm{mmhg}$, the respiratory frequency was primarily increased by 2 units every five minutes in both groups. In this increase, the frequency was accepted as the upper limit of 18 breaths/minute. If the $\mathrm{EtCO}_{2}$ values did not decrease under $35 \mathrm{mmHg}$ at the 5 th minute after reaching 18 breaths per minute, the $P_{\text {insp }}$ value of the patients in the $P$ group was increased by $2 \mathrm{~cm} / \mathrm{H} 2 \mathrm{O}$ every five minutes as needed. In the $V$ group, the volume settings were increased by $1 \mathrm{ml} / \mathrm{kg}$ every five minutes as needed. The upper limit was determined as $30 \mathrm{~cm} / \mathrm{H} 2 \mathrm{O}$ for the $\mathrm{P}$ group and $10 \mathrm{ml} / \mathrm{kg}$ for the $V$ group. Patients whose $\mathrm{CO}_{2}$ values did not decrease under $35 \mathrm{mmhg}$ despite mechanical ventilation with all these upper limit values were excluded from the study by making more complicated changes in mechanical ventilation and insufflation pressures. If $\mathrm{EtCO}_{2}$ values were below $33 \mathrm{mmHg}$, in both groups, it was first reduced to 10 breaths/min, and if there was no increase after five minutes, $P_{\text {insp }}$ values were decreased by $2 \mathrm{~cm} / \mathrm{H} 2 \mathrm{O}$ every five minutes in the P group, while the tidal volume was decreased by $1 \mathrm{ml} / \mathrm{kg}$ in the $V$ group. However, tidal volume was not allowed to fall below $6 \mathrm{ml} / \mathrm{kg}$ in both groups.

Demographic data (gender, age, height, weight, and ASA score) as well as operative data (anesthesia, operation, and insufflation duration) were recorded in both groups, T0 was defined as T0 before 
anesthesia, T1 after intubation, T2 5 minutes after insufflation, T3 just before desufflation, and T4 5 minutes after desufflation. Heart rate, systolic/diastolic arterial pressure values, saturation of pulseoximetry $\left(\mathrm{SpO}_{2}\right)$ and NIRS values were recorded at all time points. Additionally, $\mathrm{EtCO}_{2}$ in $\mathrm{T} 1, \mathrm{~T} 2, \mathrm{~T} 3$ and $\mathrm{T} 4$; arterial blood gas results for $\mathrm{pH}, \mathrm{pO}_{2}, \mathrm{pCO}_{2}, \mathrm{HCO}_{3}, \mathrm{BE}$ and Lactate; Tidal volume, respiratory frequency, peak pressure $\left(P_{\text {peak }}\right)$, plateau pressure $\left(P_{\text {plateau }}\right)$ and, PEEP was recorded.

\section{Statistical analysis}

SPSS 15.0 for Windows program was used for statistical analysis. Descriptive statistics; numbers and percentages for categorical variables, mean, standard deviation, minimum and maximum for numerical variables were given. Comparisons of numerical variables in two independent groups were made using the Student t-Test (when the normal distribution exists), the Mann Whitney $U$ test (when the normal distribution condition was not exist). The rates in the groups were compared with Chi-Square Analysis. Statistical alpha significance level was accepted as $p<0.05$.

\section{Results}

CONSORT diagram of the study was presented in figure 1. In total 70 patients were evaluated in the study between March and July 2020. Groups did not differ for age, BMI, operative time, anesthesia duration and insufflation duration ( $p>0.05$ for all comparisons) (Table 1 ).

Table 1

Comparison of demographic characteristics, operative time, and anesthesia duration of the patients that underwent volume controlled/pressure controlled ventilation in laparoscopic cholecystectomy

\begin{tabular}{|c|c|c|c|c|}
\hline & & Grup V & Grup P & \\
\hline & & $\mathrm{n}=35(\%)$ & $\mathrm{n}=35(\%)$ & $\mathrm{p}$ \\
\hline Gender & Male & $7(20,0)$ & $11(31,4)$ & ${ }^{b} 0,274$ \\
\hline & Female & $28(80,0)$ & $24(68,6)$ & \\
\hline & & Mean. $\pm S D($ Min-Max) & Ort. $\pm S D$ (Min-Max) & $\mathrm{p}$ \\
\hline Age & & $48,8 \pm 11,1(27-65)$ & $48,0 \pm 10,1(23-65)$ & ${ }^{a} 0,753$ \\
\hline BMI & & $27,1 \pm 2,3(20-30)$ & $26,7 \pm 3,0(20-30)$ & ${ }^{c} 0,882$ \\
\hline Operativ & & $76,0 \pm 16,8(50-110)$ & $77,3 \pm 19,9(50-130)$ & ${ }^{c} 0,990$ \\
\hline Anesthes & duration & $86,0 \pm 16,8(60-120)$ & $87,6 \pm 20,2(60-140)$ & ${ }^{c} 0,952$ \\
\hline İnsufflati & duration & $41,4 \pm 9,4(30-70)$ & $40,0 \pm 11,8(30-90)$ & $c^{c} 0,297$ \\
\hline
\end{tabular}


No significant difference exists in the right and left averages of T0 and T4 NIRS measurements in both groups ( $p=0.060 p=0.208)$. Mean values of NIRS at right T1-2-3 and left T2-3 of Group P were significantly higher than Group $\vee(p=0.030 p=0.001 p=0.001 p=0.006 p=0.002$ respectively) (Table 2).

Table 2

Comparison of NIRS of patients that underwent volume controlled/pressure controlled ventilation in laparoscopic cholecystectomy

\begin{tabular}{|c|c|c|c|c|c|c|}
\hline & & \multicolumn{2}{|l|}{ Grup V } & \multicolumn{3}{|l|}{ Grup P } \\
\hline & & Mean $\pm S D$ & Median (Min-Max) & Mean $\pm S D$ & Median (Min-Max) & $p$ \\
\hline \multirow[t]{5}{*}{ NIRS-Right } & T0 & $65,5 \pm 4,8$ & $65(54-75)$ & $67,7 \pm 5,0$ & 68 (57-79) & ${ }^{a} 0,060$ \\
\hline & $\mathrm{T} 1$ & $67,1 \pm 6,5$ & $67(52-80)$ & $70,5 \pm 6,1$ & $71(58-80)$ & ${ }^{a} 0,030$ * \\
\hline & T2 & $64,8 \pm 5,9$ & 65 (54-75) & $70,1 \pm 6,4$ & $72(56-81)$ & ${ }^{a} 0,001$ * \\
\hline & T3 & $67,4 \pm 5,0$ & 68 (58-77) & $71,7 \pm 4,9$ & $73(62-82)$ & ${ }^{a} 0,001$ * \\
\hline & T4 & $68,1 \pm 4,5$ & 69 (59-77) & $70,1 \pm 5,0$ & $70(62-83)$ & ${ }^{a} 0,081$ \\
\hline \multirow[t]{5}{*}{ NIRS-Left } & T0 & $65,0 \pm 4,6$ & $65(57-77)$ & $66,5 \pm 5,5$ & $66(55-80)$ & ${ }^{a} 0,208$ \\
\hline & $\mathrm{T} 1$ & $66,2 \pm 6,7$ & $66(54-85)$ & $68,7 \pm 6,6$ & $68(55-81)$ & ${ }^{a} 0,122$ \\
\hline & T2 & $64,1 \pm 6,5$ & $63(53-77)$ & $68,4 \pm 6,1$ & 69 (53-79) & ${ }^{a} 0,006 *$ \\
\hline & T3 & $65,9 \pm 5,2$ & $67(55-74)$ & $70,2 \pm 6,0$ & $71(56-81)$ & ${ }^{a} 0,002^{*}$ \\
\hline & T4 & $66,7 \pm 4,9$ & 68 (56-77) & $68,7 \pm 5,6$ & $69(57-80)$ & ${ }^{c} 0,137$ \\
\hline
\end{tabular}

Hemodynamic parameters were presented in Table 3. The systolic, diastolic and, mean arterial pressures did not differ between groups ( $p>0.05$ for all comparisons). The heart rate at T0, T2 and T3 was significantly high in group $P\left(p=0,017 p=0,043 p=0,020\right.$ respectively). The $\mathrm{SpO}_{2}$ levels was significantly lower in group $P$ at $\mathrm{TO}(\mathrm{p}=0,006)$. The $\mathrm{EtCO}_{2}$ levels was significantly higher in group $\mathrm{P}$ at $\mathrm{T} 2$ time point $(p=0,008)$. Other comparisons for hemodynamic parameters were not significant. The comparisons for blood gas parameters were not significant as well ( $p>0.05$ for all comparisons) (Table 4). 
Table 3

Hemodynamic parameters of the patients that underwent volume controlled/pressure controlled ventilation in laparoscopic cholecystectomy

\begin{tabular}{|c|c|c|c|c|c|c|}
\hline & & \multicolumn{2}{|l|}{ Grup V } & \multicolumn{3}{|l|}{ Grup P } \\
\hline & & Mean $\pm S D$ & Median (Min-Max) & Mean. $\pm S D$ & Median (Min-Max) & $\mathrm{p}$ \\
\hline \multirow[t]{5}{*}{ SAP } & TO & $141,5 \pm 15,1$ & $140(108-170)$ & $135,4 \pm 23,7$ & 139 (30-180) & ${ }^{a} 0,204$ \\
\hline & T1 & $115,6 \pm 20,5$ & $110(90-167)$ & $109,1 \pm 12,9$ & 107 (90-137) & ${ }^{a} 0,121$ \\
\hline & $\mathrm{T} 2$ & $118,2 \pm 18,2$ & $120(85-174)$ & $119,1 \pm 18,6$ & $116(90-167)$ & ${ }^{a} 0,826$ \\
\hline & T3 & $114,1 \pm 13,5$ & $110(90-149)$ & $108,7 \pm 21,4$ & $108(20-151)$ & ${ }^{c} 0,206$ \\
\hline & $\mathrm{T} 4$ & $110,0 \pm 14,4$ & 107 (86-148) & $110,2 \pm 10,9$ & $112(83-130)$ & ${ }^{a} 0,940$ \\
\hline \multirow[t]{5}{*}{ DAP } & T0 & $82,9 \pm 13,7$ & $81(60-114)$ & $82,2 \pm 9,7$ & $85(58-100)$ & ${ }^{c} 0,809$ \\
\hline & T1 & $69,5 \pm 16,6$ & $70(8-110)$ & $69,3 \pm 9,5$ & $70(52-86)$ & ${ }^{a} 0,930$ \\
\hline & $\mathrm{T} 2$ & $75,4 \pm 12,0$ & $70(47-102)$ & $78,3 \pm 13,6$ & $80(50-106)$ & ${ }^{a} 0,356$ \\
\hline & T3 & $69,2 \pm 11,4$ & $69(49-102)$ & $67,8 \pm 12,5$ & $66(45-91)$ & ${ }^{a} 0,612$ \\
\hline & $\mathrm{T} 4$ & $67,1 \pm 11,1$ & $67(50-90)$ & $67,5 \pm 8,7$ & $68(50-80)$ & ${ }^{a} 0,886$ \\
\hline \multirow[t]{5}{*}{ MAP } & T0 & $107,1 \pm 11,8$ & 105 (80-132) & $104,2 \pm 11,8$ & $106(79-128)$ & ${ }^{a} 0,311$ \\
\hline & T1 & $89,9 \pm 15,2$ & $86(68-132)$ & $86,3 \pm 9,5$ & $86(71-106)$ & ${ }^{a} 0,240$ \\
\hline & $\mathrm{T} 2$ & $90,5 \pm 12,6$ & $91(64-112)$ & $95,1 \pm 15,4$ & $94(70-132)$ & ${ }^{a} 0,181$ \\
\hline & T3 & $88,2 \pm 11,8$ & $85(73-123)$ & $86,8 \pm 12,7$ & $86(68-118)$ & ${ }^{c} 0,724$ \\
\hline & $\mathrm{T} 4$ & $86,1 \pm 13,2$ & $85(65-123)$ & $86,5 \pm 10,1$ & $89(62-103)$ & ${ }^{a} 0,879$ \\
\hline \multirow[t]{5}{*}{ HR } & T0 & $76,5 \pm 11,2$ & $76(61-100)$ & $84,2 \pm 14,8$ & $85(54-120)$ & ${ }^{a} 0,017$ * \\
\hline & T1 & $77,9 \pm 12,2$ & $80(57-100)$ & $82,8 \pm 14,2$ & $81(63-120)$ & ${ }^{a} 0,125$ \\
\hline & $\mathrm{T} 2$ & $68,3 \pm 10,7$ & $69(50-88)$ & $74,6 \pm 14,7$ & $72(50-119)$ & ${ }^{a} \mathbf{0 , 0 4 3}$ * \\
\hline & T3 & $62,2 \pm 10,0$ & $61(45-90)$ & $68,0 \pm 10,4$ & $69(49-96)$ & ${ }^{a} 0,020$ * \\
\hline & $\mathrm{T} 4$ & $65,3 \pm 12,3$ & $64(50-108)$ & $62,7 \pm 10,7$ & $60(45-87)$ & ${ }^{c} 0,424$ \\
\hline
\end{tabular}

${ }^{a}$ Student-t Test, ${ }^{c}$ Mann Whitney U Test, ${ }^{*} p<0,05$, SAP: Systolic arterieal pressure, DAP: Diastolic arterial pressure, MAP: Mean arterial pressure, HR: Heart Rare, ETCO 2 End Tidal $\mathrm{CO}_{2}$ 


\begin{tabular}{|c|c|c|c|c|c|c|}
\hline & & Grup V & & Grup P & & \\
\hline \multirow[t]{5}{*}{$\mathrm{SPO}_{2}$} & T0 & $98,5 \pm 1,3$ & $99(95-100)$ & $98,7 \pm 1,3$ & $99(96-100)$ & ${ }^{c} 0,456$ \\
\hline & T1 & $99,1 \pm 0,9$ & $99(97-100)$ & $98,4 \pm 1,0$ & $98(96-100)$ & ${ }^{c} 0,006 *$ \\
\hline & T2 & $98,4 \pm 2,6$ & $99(85-100)$ & $98,3 \pm 1,5$ & $98(93-100)$ & ${ }^{c} 0,314$ \\
\hline & T3 & $98,8 \pm 1,1$ & $99(97-100)$ & $98,7 \pm 1,2$ & $99(96-100)$ & ${ }^{c} 0,710$ \\
\hline & $\mathrm{T} 4$ & $99,2 \pm 0,9$ & $99(97-100)$ & $98,7 \pm 1,1$ & $99(96-100)$ & $c^{c} 0,113$ \\
\hline \multirow[t]{4}{*}{$\mathrm{ETCO}_{2}$} & T1 & $32,2 \pm 2,4$ & $32(27-36)$ & $32,6 \pm 1,7$ & $33(28-35)$ & ${ }^{c} 0,454$ \\
\hline & $\mathrm{T} 2$ & $33,1 \pm 2,8$ & $33(25-40)$ & $34,5 \pm 1,7$ & 35 (29-38) & ${ }^{c} 0,008$ * \\
\hline & T3 & $34,5 \pm 1,3$ & $35(32-38)$ & $34,0 \pm 2,6$ & $34(26-43)$ & ${ }^{c} 0,194$ \\
\hline & T4 & $33,6 \pm 1,8$ & $34(29-38)$ & $33,5 \pm 1,6$ & $34(30-38)$ & $c^{c} 0,692$ \\
\hline
\end{tabular}


Table 4

Blood gas parameters of the patients that underwent volume controlled/pressure controlled ventilation in laparoscopic cholecystectomy

\begin{tabular}{|c|c|c|c|c|c|c|}
\hline & & Grup V & Grup P & & & \\
\hline & & Mean. $\pm S D$ & Median (Min-Max) & Ort. $\pm S D$ & Median (Min-Max) & $\mathrm{p}$ \\
\hline \multirow[t]{4}{*}{$\mathrm{PH}$} & T1 & $7,45 \pm 0,04$ & $7,45(7,38-7,52)$ & $7,43 \pm 0,04$ & 7,43 (7,33-7,49) & ${ }^{a} 0,118$ \\
\hline & T2 & $7,41 \pm 0,04$ & 7,41 (7,33-7,49) & $7,40 \pm 0,04$ & $7,41(7,3-7,46)$ & ${ }^{a} 0,273$ \\
\hline & T3 & $7,40 \pm 0,04$ & $7,40(7,33-7,50)$ & $7,41 \pm 0,04$ & $7,42(7,3-7,49)$ & ${ }^{c} 0,285$ \\
\hline & T4 & $7,39 \pm 0,04$ & 7,38 (7,31-7,47) & $7,40 \pm 0,04$ & $7,41(7,3-7,49)$ & ${ }^{a} 0,161$ \\
\hline \multirow[t]{5}{*}{$\mathrm{PO}_{2}$} & T1 & $157,2 \pm 26,6$ & $150(118-220)$ & $159,7 \pm 32,9$ & $160(90-220)$ & ${ }^{a} 0,730$ \\
\hline & T2 & $140,3 \pm 27,4$ & 138 (95-190) & $144,9 \pm 30,5$ & $140(95-200)$ & ${ }^{c} 0,510$ \\
\hline & T3 & $142,4 \pm 34,0$ & $140(90-263)$ & $151,7 \pm 25,3$ & $150(109-210)$ & ${ }^{a} 0,195$ \\
\hline & T4 & $163,2 \pm 44,2$ & $150(107-355)$ & $161,0 \pm 30,5$ & $150(115-260)$ & ${ }^{c} 0,750$ \\
\hline & T4 & $-0,15 \pm 1,68$ & $0,2(-4-2,6)$ & $-0,73 \pm 1,95$ & $-0,8(-5,3-2,7)$ & ${ }^{c} 0,172$ \\
\hline \multirow[t]{4}{*}{$\mathrm{PCO}_{2}$} & T1 & $35,1 \pm 2,9$ & $35(30-40)$ & $35,8 \pm 2,1$ & $36(32-41)$ & ${ }^{c} 0,280$ \\
\hline & T2 & $37,7 \pm 2,8$ & $38(32-43)$ & $38,2 \pm 1,9$ & $38(34-41)$ & ${ }^{a} 0,503$ \\
\hline & T3 & $38,3 \pm 2,9$ & $38(32,6-43)$ & $37,3 \pm 2,5$ & $38(30-43)$ & ${ }^{c} 0,166$ \\
\hline & T4 & $38,9 \pm 4,1$ & $38(34-53)$ & $37,1 \pm 2,4$ & $37(32-42)$ & ${ }^{c} 0,116$ \\
\hline \multirow[t]{4}{*}{$\mathrm{HCO}_{3}$} & $\mathrm{~T} 1$ & $24,9 \pm 1,8$ & $25,5(20-27,6)$ & $24,3 \pm 1,7$ & $25(18-26,6)$ & ${ }^{c} 0,111$ \\
\hline & T2 & $24,3 \pm 2,0$ & $25(20-27,5)$ & $23,9 \pm 1,6$ & $24(20-26,8)$ & ${ }^{c} 0,124$ \\
\hline & T3 & $24,1 \pm 1,9$ & $25(20-27)$ & $23,6 \pm 1,8$ & $24(20-27,4)$ & ${ }^{c} 0,083$ \\
\hline & T4 & $24,0 \pm 1,6$ & $24(21-26,2)$ & $23,6 \pm 1,8$ & $24(20-26,9)$ & ${ }^{c} 0,284$ \\
\hline \multirow[t]{4}{*}{ BE } & T1 & $0,71 \pm 1,82$ & $1,5(-3,4-3,6)$ & $0,04 \pm 1,89$ & $0,5(-7-2,5)$ & ${ }^{c} 0,052$ \\
\hline & $\mathrm{T} 2$ & $0,20 \pm 1,96$ & $0,9(-4-3,4)$ & $-0,47 \pm 1,84$ & $-0,2(-5,5-2,4)$ & ${ }^{c} 0,071$ \\
\hline & T3 & $0,03 \pm 1,84$ & $0,5(-4-2,9)$ & $-0,57 \pm 2,11$ & $-0,3(-5,5-3,3)$ & ${ }^{c} 0,115$ \\
\hline & T4 & $-0,15 \pm 1,68$ & $0,2(-4-2,6)$ & $-0,73 \pm 1,95$ & $-0,8(-5,3-2,7)$ & $c^{c} 0,172$ \\
\hline
\end{tabular}




\begin{tabular}{|c|c|c|c|c|c|c|}
\hline & & Grup V & & & & \\
\hline Lactate & $\mathrm{T} 1$ & $1,30 \pm 0,46$ & $1,1(0,6-2,3)$ & $1,27 \pm 0,63$ & $1,2(0,4-2,8)$ & ${ }^{c} 0,432$ \\
\hline \multirow[t]{3}{*}{$(\mathrm{mmol} / \mathrm{L})$} & $\mathrm{T} 2$ & $1,51 \pm 0,57$ & $1,4(0,8-2,6)$ & $1,49 \pm 0,65$ & $1,2(0,7-3,1)$ & ${ }^{c} 0,786$ \\
\hline & T3 & $1,53 \pm 0,69$ & $1,3(0,5-3,5)$ & $1,47 \pm 0,55$ & $1,3(0,7-3,1)$ & ${ }^{c} 0,934$ \\
\hline & $\mathrm{T} 4$ & $1,41 \pm 0,59$ & $1,3(0,5-3)$ & $1,37 \pm 0,64$ & $1,2(0,5-3,2)$ & ${ }^{c} 0,736$ \\
\hline
\end{tabular}

For ventilation parameters, $\mathrm{P}_{\text {peak }}$ and $\mathrm{P}_{\text {plateau }}$ levels in $\mathrm{T} 1, \mathrm{~T} 2$ and $\mathrm{T} 4$ were significantly lower in group $\mathrm{P}$ $(p=0,003 p=0,001 p<0,001 p=0,011 p=0,001 p<0,001$ respectively). The TV at T3 was significantly high in group $P(p=0,017)$. Other comparisons for ventilation parameters were not significant $(p>0.05$ for all comparisons) (Table 5). 
Table 5

Ventilation parameters that underwent volume controlled/pressure controlled ventilation in laparoscopic cholecystectomy

\begin{tabular}{|c|c|c|c|c|c|c|}
\hline & & Grup V & & Grup P & & \\
\hline & & Ort. $\pm S D$ & Median (Min-Maks) & Ort. $\pm S D$ & Median (Min-Maks) & $p$ \\
\hline \multirow[t]{4}{*}{$P_{\text {peak }}$} & T1 & $16,9 \pm 2,9$ & $17(13-26)$ & $15,0 \pm 2,1$ & $15(11-19)$ & ${ }^{c} 0,003^{*}$ \\
\hline & T2 & $23,1 \pm 3,8$ & $24(16-33)$ & $20,3 \pm 2,7$ & $21(15-25)$ & ${ }^{a} 0,001$ * \\
\hline & T3 & $21,6 \pm 3,7$ & $21(15-31)$ & $20,0 \pm 3,2$ & $20(14-25)$ & ${ }^{a} 0,053$ \\
\hline & $\mathrm{T} 4$ & $18,8 \pm 2,7$ & $19(14-27)$ & $15,3 \pm 2,1$ & $16(11-20)$ & $c_{<}<0,001 * *$ \\
\hline \multirow[t]{4}{*}{$P_{\text {plateau }}$} & T1 & $16,3 \pm 2,6$ & $16(12-24)$ & $14,8 \pm 2,0$ & $15(11-19)$ & ${ }^{a} 0,011$ * \\
\hline & T2 & $22,5 \pm 3,6$ & $23(16-30)$ & $19,9 \pm 2,5$ & $20(15-25)$ & ${ }^{a} 0,001$ * \\
\hline & T3 & $20,8 \pm 3,7$ & $20(15-30)$ & $19,5 \pm 3,1$ & $20(14-25)$ & ${ }^{c} 0,129$ \\
\hline & T4 & $17,8 \pm 2,5$ & $18(14-25)$ & $15,0 \pm 2,2$ & $15(11-20)$ & $a<0,001 *$ \\
\hline \multirow[t]{4}{*}{ TV } & $\mathrm{T} 1$ & $472,3 \pm 38,0$ & $500(400-550)$ & $492,5 \pm 61,9$ & $500(400-650)$ & ${ }^{c} 0,244$ \\
\hline & T2 & $470,3 \pm 37,5$ & $450(400-550)$ & $482,4 \pm 65,0$ & $500(380-600)$ & ${ }^{c} 0,380$ \\
\hline & T3 & $470,6 \pm 39,9$ & $480(400-550)$ & $508,4 \pm 70,7$ & $500(400-650)$ & ${ }^{c} 0,017^{*}$ \\
\hline & T4 & $470,9 \pm 37,8$ & $450(400-550)$ & $486,1 \pm 64,5$ & $480(400-650)$ & ${ }^{c} 0,744$ \\
\hline \multirow[t]{4}{*}{$f$} & $\mathrm{~T} 1$ & $11,7 \pm 0,8$ & $12(10-14)$ & $11,5 \pm 1,0$ & $12(10-14)$ & ${ }^{c} 0,383$ \\
\hline & T2 & $12,2 \pm 1,6$ & $12(10-16)$ & $12,3 \pm 1,3$ & $12(10-16)$ & ${ }^{c} 0,774$ \\
\hline & T3 & $13,3 \pm 1,5$ & $14(10-16)$ & $12,9 \pm 1,1$ & $12(10-14)$ & ${ }^{c} 0,161$ \\
\hline & T4 & $13,2 \pm 1,5$ & $12(10-16)$ & $12,2 \pm 0,8$ & $12(10-14)$ & ${ }^{c} 0,078$ \\
\hline
\end{tabular}

\section{Discussion}

In a randomized controlled setting, our results indicate that cerebral oxygenation was better in patients ventilated with PCV mode due to higher NIRS values and lower $P_{\text {peak }}$ and $P_{\text {plateau }}$ values.

The laparoscopic surgery improves the quality of life by avoiding abdominal incisions, extensive dissection and related comorbidities. ${ }^{1}$ However pneumoperitoneum causes an increase in intra- 
abdominal pressure and indirectly a decrease in lung volumes, functional residual capacity and pulmonary compliance. An increase in airway resistance may be resulted with development of atelectasis in the basal parts of the lung and ventilation-perfusion mismatch can occur. ${ }^{1,3}$ The VCV mode increases $P_{\text {peak }}$ and $P_{\text {plateau }}$ values which are directly related with the lung damage. In a randomized controlled setting, Sen et al. compared VCV and PCV on 40 patients who underwent laparoscopic cholecystectomy. The results indicate that $P_{\text {peak }}$ and $P_{\text {plateau }}$ pressures were higher in patients who underwent VCV after pneumoperitoneum. ${ }^{7}$ Netthra et al. compared VCV and PCV on 60 laparoscopic cholecystectomy patients. Their results indicate that PCV resulted with lower P mean and Ppeak values. Our study is also consistent with the studies which resulted in favor of PCV in laparoscopic cholecystectomy. Our results indicate that $P_{\text {peak }}$ and $P_{\text {plateau }}$ values were found to be significantly higher in the VCV group, especially after insufflation. Literary data indicate that VCV may decrease the safety index by increasing the risk of volu-trauma and barotrauma in the VCV mode in laparoscopic cases. To stop the increase in $P_{\text {peak }}$ pressure and decrease lung injury, applications such as changing the respiratory rate and tidal volume or switching to PCV mode are performed. ${ }^{9}$ Although the PCV mode is a good method in the management of elevated $P_{\text {peak }}$ values, its effects on ventilation dynamics and hemodynamic parameters did not clearly define.

The high $\mathrm{P}_{\text {peak }}$ values in VCV mode may also resulted with decrease in partial oxygen pressure. However, the effect of VCV and PCV modes on tissue oxygenation are contradictory. Balick-Weber et al. examined the respiratory effects of laparoscopic surgery on 21 patients. No change was shown on partial oxygen pressures after insufflation. ${ }^{10}$ Hans et al. also reported no significant difference between PO2 pressures on 40 obese patients who underwent laparoscopic by-pass operation. ${ }^{11}$ However, in two other studies conducted in obese patients, partial oxygen pressures were shown to be higher in patients ventilated with PCV mode. ${ }^{12,13}$ In our study, partial oxygen pressure values were higher in PCV mode, however no significant difference was found for blood gas parameters between groups.

Tissue oxygenation measurements have been used frequently in perioperative patient management in recent years. Different methods such as bispectral index electroencephalography or auditory evoke potentials are used to measure anesthetic depth. The NIRS is another method which used to evaluate the depth of anesthesia by measuring the oxygenation change at the tissue level in the prefrontal cortex. ${ }^{14}$ We could not be able to find a study that evaluates the cerebral oxygenation with NIRS in laparoscopic surgery. However, NIRS was used in different surgeries previously.

Green et al. ${ }^{6}$ in their study with 46 patients who underwent major abdominal surgery, detected low tissue oxygenation using the NIRS method, which could not be detected by conventional monitoring methods. Gibson et al. ${ }^{15}$ compared NIRS values before and after insufflation in 70 patients who had undergone laparoscopic abdominal surgery and showed that NIRS values decreased statistically after insufflation. Although there was no significant difference between $\mathrm{SpO}_{2}$ and $\mathrm{PaO}_{2}$ pressures in our study, the NIRS values of patients who underwent PCV were found to be significantly higher during pneumoperitoneum compared to the VCV group. This may be an evidence that oxygenation disorder occurs at the tissue level, 
although the resulting oxygenation change is not reflected in conventional monitoring parameters and arterial blood gas analysis.

Kurukahvecioğlu et al. ${ }^{16}$ in their study with 60 patients who had undergone laparoscopic abdominal surgery showed that insufflation pressure caused blood to pool in the lower extremities, which decreased cerebral NIRS values. This decrease is a mechanical result of the high pressure created by insufflation in the abdomen. This mechanical condition occurs not only in the abdomen, but also in the thorax, with the high $\mathrm{P}_{\text {peak }}$ created by the VCV mode, as demonstrated in our study. Increased intrathoracic pressure reduces preload and indirectly cardiac output, and consequently explains the significantly lower NIRS values in the VCV group in our study.

The limitation of our study is that we had to use $P_{\text {peak }}$ and $P_{\text {plateau }}$ instead of trans-pulmonary pressure to evaluate the safety of controlled mechanical ventilation modes. Because transpulmonary pressure is the most objective parameter in the evaluation of ventilator induced lung injuries. However, it was not preferred because it is measured by invasive methods.

\section{Conclusion}

In laparoscopic cholecystectomy operations, tissue oxygenation with PCV mode is higher than with VCV mode. In PCV mode, the risk of lung barotrauma, which is likely due to high $P_{\text {peak }}$ and $P_{\text {plateau }}$ values, is lower. NIRS can be used in laparoscopic cholecystectomy cases because it is more sensitive, noninvasive and easy to use than arterial blood gas analysis in measuring tissue oxygenation.

\section{Abbreviations}

NIRS: near infrared spectroscopy

VCV: Volume-controlled ventilation

PCV: pressure-controlled ventilation

TV: Tidal volume

ASA: American Society of Anesthesiology

BMI: body mass index

$P_{\text {insp }}$ : inspiratory pressure

$P_{\text {peak: }}$ peak pressure

$\mathrm{P}_{\text {plateau: }}$ plateau pressure

PEEP: positive end expiratory pressure 


\section{Declarations}

Ethics and Consent to participate: Informed consent was taken from all patients. All procedures that performed in our study were made in accordance with the ethical standards of the Helsinki declaration (2008). This clinical study was approved by Sisli Hamidiye Etfal Training and Research Hospital Ethics Committee with the number 1496 on 02/06/2020.

Consent for publication: Not applicable

Availability of data and materials: The datasets used and/or analysed during the current study available from the corresponding author on reasonable request.

Competing interests: This research received no spesific grant from any funding agency in the public, commercial or no-for-profit sectors. The authors declare there are no conflicts of interests.

Funding: This research received no spesific grant from any funding agency in the public, commercial or no-for-profit sectors.

Author's contributions: Emre Badur, Mustafa Altınay, Leyla Türkoğlu and A. Surhan Çınar conceived and designed the study. Emre Badur, Mustafa Altınay, Pınar Sayın, Tuğba Yücel and Leyla Türkoğlu carried out the study protocol and retrospectively performed data collection. Emre Badur, Mustafa Altınay, and Pınar Sayın performed statistical analysis and data interpretation. All authors contributed to the writing of and approved the final manuscript.

Acknowledgements: Not applicable

\section{References}

1. Marco AP, Yeo CJ, Rock P. Anesthesia for patient undergoing laparoscopic cholecystectomy. Anesthesiology 1990;73:1268-70.

2. Cunningam AJ, Brul SJ. Laparoscopic cholecystectomy; anesthetic implications. Anesth Analg 1993;76:1120-33.

3. Sayin P, Turk HS, Altınay M, Ozkan MT, Mihmanlı M, Cınar S. Comparison of Pressure Controlled and Volume Controlled Ventilation in Morbidly Obese Patients Underwent Laparoscopic Sleeve Gastrectomy: A Randomized Controlled Trial. Turkiye Klinikleri J Med Sci. 2021;41(2):109-18

4. Oti C, Mahendran M, Sabir N. Anaesthesia for laparoscopic surgery. Br J Hosp Med (Lond) 2016; 77: 24-28.

5. Nielsen HB. Systematic review of near-infrared spectroscopy determined cerebral oxygenation during non-cardiac surgery. Front Physiol. 2014;5:93. Published 2014 Mar 17. doi:10.3389/fphys.2014.00093

6. Green DW. A retrospective study of changes in cerebral oxygenation using a cerebral oximeter in older patients undergoing prolonged major abdominal surgery. Eur J Anaesthesiol. 2007;24(3):230- 
234.

7. Sen O, Umutoglu, T., Aydın, N. et al. Effects of pressure-controlled and volume-controlled ventilation on respiratory mechanics and systemic stress response during laparoscopic cholecystectomy. SpringerPlus 5, 298 (2016).

8. Nethra SS, Nagaraja S, Sudheesh K, Duggappa DR, Sanket B. Comparison of effects of volumecontrolled and pressure-controlled mode of ventilation on endotracheal cuff pressure and respiratory mechanics in laparoscopic cholecystectomies: A randomized controlled trial. Indian J Anaesth. 2020 Oct;64(10):842-848.

9. Campbell RS, Davis BR. Pressure-controlled versus volume-controlled ventilation: does it matter? Respir Care. 2002;47(4):416-426.

10. Balick-Weber CC, Nicolas P, Hedreville-Montout M, Blanchet P, Stéphan F. Respiratory and hemodynamic effects of volume-controlled vs pressure-controlled ventilation during laparoscopy: a cross-over study with echocardiographic assessment. Br J Anaesth. 2007;99(3):429-435.

11. Hans GA, Prégaldien AA, Kaba A, Sottiaux TM, DeRoover A, Lamy ML, Joris JL. Pressure-controlled ventilation does not improve gas exchange in morbidly obese patients undergoing abdominal surgery. Obes Surg. 2008;18(1):71-76.

12. Cadi P, Guenoun T, Journois D, Chevallier JM, Diehl JL, Safran D. Pressure-controlled ventilation improves oxygenation during laparoscopic obesity surgery compared with volume-controlled ventilation. Br J Anaesth. 2008 May;100(5):709-16.

13. Movassagi R, Montazer M, Mahmoodpoor A, Fattahi V, Iranpour A, Sanaie S. Comparison of pressure vs. volume-controlled ventilation on oxygenation parameters of obese patients undergoing laparoscopic cholecystectomy. Pak J Med Sci. 2017;33(5):1117-1122.

14. Hernandez-Meza G, Izzetoglu M, Osbakken M, Green M, Izzetoglu K. Near-infrared spectroscopy for the evaluation of anesthetic depth. Biomed Res Int. 2015;2015:939418.

15. Gipson $\mathrm{CL}$, Johnson GA, Fisher R, et al. Changes in cerebral oximetry during peritoneal insufflation for laparoscopic procedures. J Minim Access Surg. 2006;2(2):67-72.

16. Kurukahvecioglu O, Sare M, Karamercan A, Gunaydin B, Anadol Z, Tezel E. Intermittent pneumatic sequential compression of the lower extremities restores the cerebral oxygen saturation during laparoscopic cholecystectomy. Surg Endosc. 2008;22(4):907-911.

\section{Figures}




\section{CONSORT}

TRANSPARENT REPORTING of TRIALS

CONSORT 2010 Flow Diagram

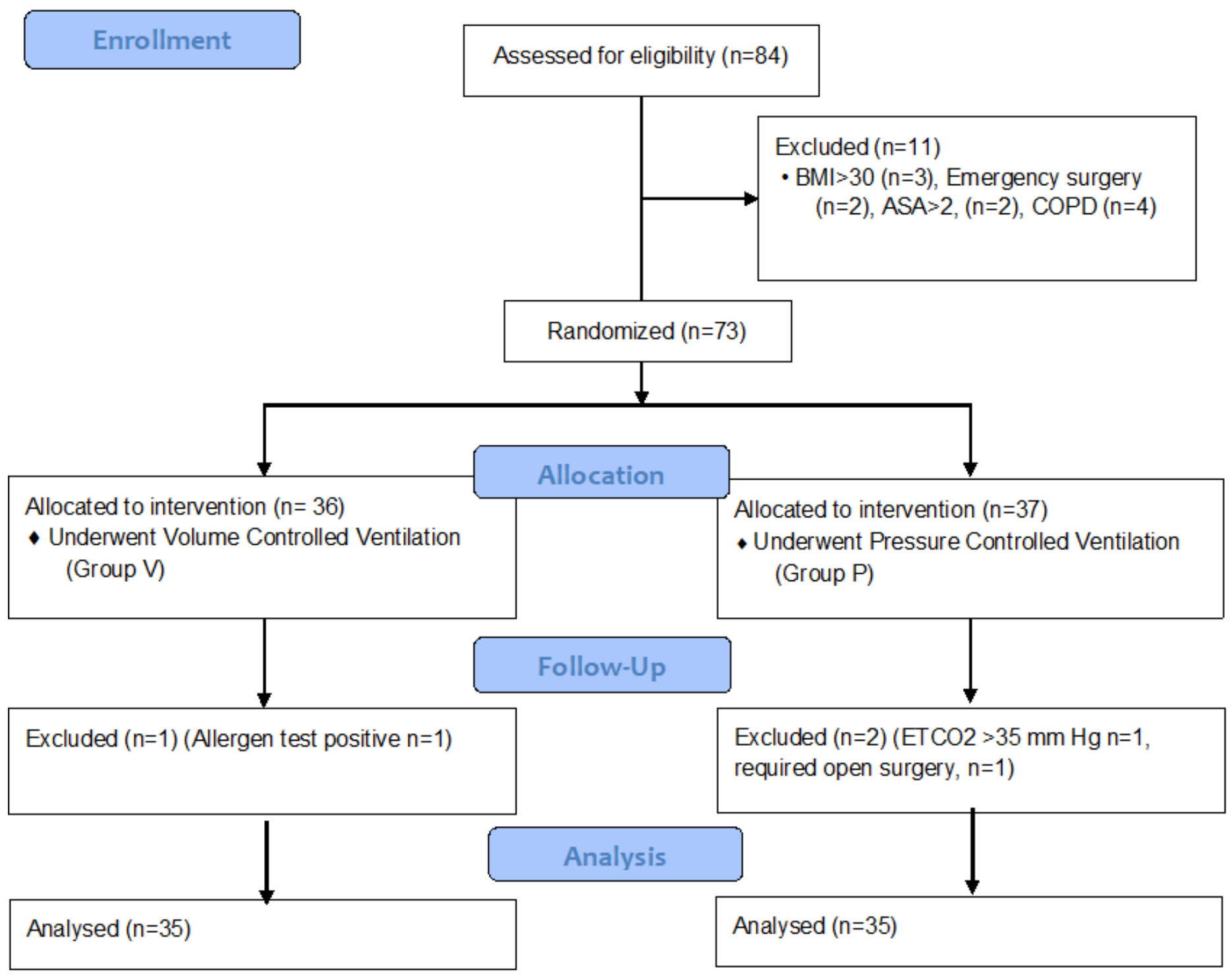

Figure 1

CONSORT diagram of the study that evaluates the effect of of Volume Controlled and Pressure Controlled Ventilation Modes on Cerebral Oxymetry and Blood Gas Status in Laparoscopıc Cholecystectomy 